Original Research Paper

\title{
Burr-X Model Estimate using Bayesian and non-Bayesian Approaches
}

\author{
Abdullah Y. Al-Hossain \\ Department of Mathematics, Faculty of Science, Jazan University, Jazan, Saudi Arabia
}

Article history

Received: 03-03-2016

Revised: 09-04-2016

Accepted: 28-04-2016

Email: aalhossain@jazanu.edu.sa

\begin{abstract}
The present paper is aimed at developing Bayesian and Maximum Likelihood estimations (ML) of the Burr type-X model of distribution when data are gathered from Type-II cumulative censoring with binomial eliminations. The procedures for getting the (ML) evaluations of the parameters are examined. The Bayes technique to get both point and interval estimators of the parameters are illustrated. The expected termination time for Type-II cumulative censoring with binomial eliminations is analyzed after carrying out the computation. Classical and Bayes procedures are improved in the case of parameter estimation and evaluated the expected test time for Burr-X model under cumulative censoring wit binomial sweep. A simulation study is performed to compare the implementation of the various procedures and for the expected termination time of the test. Finally, illustrative examples are given and the results from emulation studies determining the achievement of the suggested techniques are presented.
\end{abstract}

Keywords: Burr Type-X Model, Maximum Likelihood Estimator, Bayes Estimator, Life Testing, Expected Duration

\section{Introduction}

Censoring is normal in life-distribution research due to limits and other constraints on data gathering. Censoring happens when exact lifetimes are recognized only for a part of the individuals or units under investigating while for the rest of the lifetimes data on them is partial. It is known that the most common censored tests are type-II censoring for reserving money and time. For more details about the progressive censoring schemes and applications, we refer to the monograph Balakrishnan and Aggarwala (2000). The assessment of parameters from various lifetime models in the context of cumulative Type-II censored samples have been considered by many authors including Balakrishnan et al. (2003; Fernandez, 2004; Ng et al., 2004; Soliman, 2005; Wu et al., 2007; Ku and Kaya, 2007; Banerjee and Kundu (2008). However, there is some work presented in the Bayesian model. Amin (2008) expanded Bayesian techniques in the case of parameter appreciation and assessment of future surveillances from the classical Pareto model. In Bayesian framework, many authors, see among others, Zellner (1995; Torney, 2005; Yan et al., 2009; Naranjo et al., 2014) had done some work. For some related classical estimation on cumulative Type-II censoring with random cancellations or binomial eliminations, one may refer to Tse et al. (2000; Wu, 2003). Also, we may mention the following related researchers for different hypotheses: Cohen (1963) illustrated the gradually censored specimens in life testing. Efron (1982) studied the bootstrap and other resembling plans. Balakrishnan and Sandu (1995) gave a simple computation algorithm for generating gradually Type-II censored specimens. Soliman et al. (2011; Mahmoud et al., 2013) discussed Bayesian inference and expectation of Burr types model for cumulative first failure censored pattern. Al-Hossain (2015a) considered generalized and inverse Gaussian distributions for the power inverse Gaussian. Al-Hossain (2016) studied the inference from the Exponentiated Weibull distribution given adaptive cumulative censored information. Sutikno and Ratih (2014) presented the Gaussian Copula Marginal degradation for design maximum information with implementation.

Recently, many appreciation issues for the Burr-X model Bayesian and non-Bayesian approaches, when the duration of life is gathered in the case of Type-II cumulative censoring, where the number of units taken 
away at each failure time obeys a binomial model of distribution is illustrated. For example, Clifford et al. (2015) proposed a Bayesian approach to person perception which outlining the theoretical position and a methodological framework for testing the predictions experimentally. Ahmad et al. (2015) obtained a Bayes estimates of the parameters and MLE for Burr-X model in the case of double type-II censored specimen of dual generalized order statistics. AL-Hussaini et al. (2014) studied the Bayesian expectation bounds of order statistics established on gradually type-II censored competing risks information from a common class of distributions. Ali (2014) presented a mixture of the inverse Rayleigh distribution with properties and estimation in a Bayesian framework. Okasha and Matter (2015) discussed more parameters burr-XII model and its application to heavy tailed lifetime information. Silva and Cordeiro (2015) introduced a new family of distributions by compounding the Burr-XII and power series distributions. Wang and Lee (2014) displayed the M-estimator procedure founded on the Bisquare objective function to evaluate the Burr-III parameters. Okasha (2014) considered the E-Bayesian method for computing estimates of the unknown parameter and some survival time parameters e.g., hazard and reliability functions of Lomax model in the case of type-II censored information. Pathak and Chaturvedi (2014) derived an Estimation of the reliability function for two-parameter exponentiated Rayleigh or Burr type X distribution. Karam and Jbur (2014) offered Bayesian analyzes of the Burr type distribution under double type censored samples using different priors and loss functions. Furthermore, a short version of this paper has been previously published in conference proceedings (Al-Hoaasin, 2015b).

In this study, classical and Bayesian techniques are expanded in the case of parameter assessment and evaluated the expected test time for Burr-X distribution under cumulative censoring with binomial eliminations. Finally, this paper is arranged as the model formulation of the problem is given in section 2. In section 3, the techniques for finding the (MLE) of the parameters $\theta$ and $p$ are presented. Interval estimations of the parameters and both point are obtained. Bayesian techniques are using to illustrate point and interval estimations in sections 4 . In section 5 , the prospective duration in the case of Type-II cumulative censoring with the influence of different $p$ is examined. Illustrative examples and the outcomes from emulation studies imposing the implementation of our suggested technique have been shown in section 6 . In the end, we conclude the work in section 7 .

\section{Model Description}

Assume the lifetime of a specific unit has a Burr-X model with probability density function (pdf), the corresponding cumulative distribution function (cdf) and the conditional likelihood function may be expressed as in Al-Hossain (2015b):

$$
\begin{aligned}
& f(x, \theta)=2 \theta x \exp \left(-x^{2}\right)\left(1-\exp \left(-x^{2}\right)\right)^{\theta-1} \\
& F_{X}(x)=\left(1-\exp \left(-x^{2}\right)\right)^{\theta} \\
& L(\theta, x \mid R=r)=c \prod_{i=1}^{m} f\left(x_{i}\right)\left[1-F\left(x_{i}\right)\right]^{r_{i}}
\end{aligned}
$$

where, $x, \theta>0$. For more details see Al-Hossain (2015b).

From (1) and (2) into (3), we obtain:

$$
\begin{aligned}
& L(\theta, x \mid R=r)=c T(x) \theta^{m} \sum_{j=0}^{n} \cdots \sum_{j_{m}=0}^{r_{m}} G \\
& \times \exp \left(\theta \sum_{i=1}^{m}(j i+1) \ln U_{i}\right)
\end{aligned}
$$

Assume that a single unit being taken away from the test at the $i$ th failure, $i=1,2, \cdots, m-1$ is independent of the others but with same probability $p$. After that, the number $R_{i}$ of units extracted at the $i$ th failure, $i=1,2, \cdots$, $m-1$, follows a binomial distribution with parameters $n-m-\sum_{l=1}^{i-1} r_{l}$ and $p$. Therefore:

$P\left(R_{1}, r_{1}\right)=\left(\begin{array}{c}n-m \\ r_{1}\end{array}\right) p^{r_{1}}(1-p)^{n-m-r_{1}}$

with $i=1,2,3, \ldots, m-1$ and where $0 \leq r_{1} \leq \mathrm{n}-\mathrm{m}$ and $0 \leq r_{i}$ $\leq n-m-\sum_{l-1}^{i-1} r_{l}$ for $i=1,2,3, \cdots, m-1$.

Moreover, let that $R_{i}$ is independent of $x_{i}$ for all $i$. Then the likelihood function becomes:

$$
\begin{aligned}
& L(\theta, p ; x, r)=L(\theta ; x \mid R=r) P(R=r) \\
& P(R=r)=\frac{(n-m) !}{\left(n-m-\sum_{l=1}^{m-1} r_{i}\right) ! \prod_{i=1}^{m-1} r_{i} !}
\end{aligned}
$$

Employing (4), (6) and (7), the full likelihood function is given by:

$L(\theta, p ; x, r)=c^{*} T(x) L_{1}(\theta) L_{2}(p)$

Where:

$$
L_{1}(\theta)=\theta^{m} \sum_{j_{1}=0}^{r_{1}} \cdots \sum_{j_{m}=0}^{r_{m}} G \exp \left(\theta \sum_{i=1}^{m}\left(j_{i}+1\right) \ln U_{i}\right)
$$


And:

$$
L_{2}(p)=p \sum_{l=1}^{m-1} r_{i}(1-p)^{(m-1)(n-m)}-\sum_{l=1}^{m-1}(m-i) r_{i},
$$

With:

$$
c^{*}=\frac{c(n-m) !}{\left(n-m-\sum_{l=1}^{m-1} r_{i}\right) ! \prod_{i=1}^{m-1} r_{i} !}
$$

And:

$$
T(x)=\prod_{i=1}^{m} 2 x_{i} \exp \left(-x_{i}^{2}\right)\left(U_{i}\right)^{-1}
$$

It is easy to see that both $c^{*}$ and $T(x)$ is independent of $\theta$ and $p$. For more details see Al-Hossain (2015b).

\section{Maximum Likelihood Estimation}

The methods for getting the MLE's of the parameters $\theta$ and $p$ in the context of gradually Type-II censoring information with binomial eliminations are considered. Interval and both point estimations of the parameters are obtained.

\section{Point Estimation}

The functions $L_{1}$ and $L_{2}$ are estimators of $\mathrm{p}$ and $\theta$ respectively. Consequently, the (MLE) of $\theta$ may be written as maximizing Equation 9 directly due to $L_{1}$ in Equation 9 does not depend on $p$. Similarly, $L_{2}$ in Equation 10 independent of $\theta$ so the MLE of p may be gotten directly by maximizing Equation 10 . Therefore, by considering the logarithms of $L_{1}(\theta)$ and $L_{2}(p)$, the MLE's of $\theta$ and $p$ may be obtained as in Al-Hossain (2015b).

\section{Interval Estimation}

\section{Bootstrap Confidence Intervals}

Here, the parametric bootstrap percentile technique assumed by Efron (1982) to build confidence intervals for the parameters is used. For more details see (Al-Hossain, 2015b).

\section{Approximate Interval Estimation}

In this section, the MLEs of $\theta$ and $p$ based on the approximate confidence intervals under asymptotic distributions are presented. Also, the MLEs of the parameters $\theta$ and $p$, in the case of the asymptotic variances and covariances are obtained considering the elements of the inverse of Fisher data matrix:

$$
I_{i j}=E\left[-\frac{\partial^{2} L}{\partial \theta \partial p}\right] ; i, j=1,2
$$

The approximate (observed) asymptotic variance covariance matrix for the MLEs can be derived by neglecting the anticipation factor $E$. Then we have:

$\left[\begin{array}{cc}-\frac{\partial^{2} L}{\partial \theta^{2}} & -\frac{\partial^{2} L}{\partial \theta \partial p} \\ -\frac{\partial^{2} L}{\partial p \partial \theta} & -\frac{\partial^{2} L}{\partial p^{2}}\end{array}\right]_{(\hat{\theta}, \hat{p})}^{-1}=\left[\begin{array}{cc}\operatorname{var}(\hat{\theta}) & \operatorname{cov}(\hat{\theta}, \hat{p}) \\ \operatorname{cov}(\hat{\theta}, \hat{p}) & \operatorname{var}(\hat{p})\end{array}\right]$

With:

$\frac{\partial^{2} L}{\partial \theta^{2}}=-\frac{m}{\theta^{2}}, \frac{\partial^{2} L}{\partial \theta \partial p}=\frac{\partial^{2} L}{\partial p \partial \theta}=0$

$\frac{\partial^{2} L}{\partial p^{2}} \equiv \frac{\sum_{l=1}^{m-1} r_{i}}{p^{2}}+\frac{(m-1)(n-m)-\sum_{l=1}^{m-1}(m-i) r_{i}}{(1-p)^{2}}$

To compute the approximate confidence intervals for $\theta$ and $\mathrm{p}$, so the asymptotic normality of the MLE may be used. Then, (1- $\delta$ ) $100 \%$ approximate confidence intervals for $\theta$ and $p$ yield:

$\hat{\theta} \pm Z_{\delta / 2} \sqrt{\operatorname{var}(\hat{\theta})}$ and $\hat{p} \pm Z_{\delta / 2} \sqrt{\operatorname{var}(\hat{p})}$

where, $Z_{\delta / 2}$ is the percentile of the standard normal distribution with right-tail probability $\delta / 2$.

\section{Bayes Estimation}

The choice of the Loss Function (LF) is determining by an integral part of Bayesian estimation methods. In literature, many various kinds of loss functions have been considered to describe different types of loss structures. The symmetric Square Error Loss (SEL) is one of the most common loss functions. A loss function should display the consequences of various errors. Many asymmetric (LFs) may exist in the literature, however, LINEX Loss Function (LLF) is excessively utilized due to a natural expansion of SEL. Mathematically representation of LLF can be written as:

$L(\Delta) \propto e^{c \Delta}-c \Delta-1 ; \quad c \neq 0$

where, $\Delta=(\tilde{u}-u), \tilde{u}$ is an estimate of $u$.

It is simple to prove that the value of $\tilde{u}$ that minimizes $E_{u}(L(\tilde{u}-u))$ in Equation 24 is:

$\tilde{u} B L=-\frac{1}{c} \log \left(E_{u}[\exp (-c u)]\right)$

One more advantageous of asymmetric loss function is the General Entropy (GE) loss: 


$$
L_{2}(\tilde{u}-u) \propto(\tilde{u} / u)^{q}-q \log (\tilde{u} / u)-1
$$

where, the lower value happens at $\tilde{u}=u$ and the Bayes expectation value $\tilde{u}_{B G}$ of $u$ is:

$$
\tilde{u}_{B G}=\left(E_{u}\left[u^{-q}\right]\right)^{-1 / q}
$$

\section{Under GE loss (Soliman, 2005).}

\section{Point Estimation}

In this section, due to $\theta$ and $p$ considered as independent random variables, we use the gamma prior distribution with the parameters $\alpha, \beta$ for $\theta$, in some equations which are found in (Al-Hossain, 2015b).

\section{Symmetric Bayes Estimation}

Square-error loss function is so-called briefly as SEL function. The estimator of this function is the posterior mean. So, applying of the posterior densities and the Bayes estimators of the variables $\theta$ and $p$ are given in (Al-Hossain, 2015b).

\section{Asymmetric Bayes Estimation}

LINEX loss function if replacing u by $\theta$ and then $\theta$ by $u$, respectively, the Bayes estimate $\tilde{\theta}_{B L}$ of the variables $\theta$ and $p$ comparative to LINEX loss function may be expressed as:

$$
\begin{aligned}
& \tilde{\theta}_{B L}=-\frac{1}{a} \log \left[\frac{\sum_{j_{1}=0}^{r_{1}} \cdots \sum_{j_{m}=0}^{r_{m}} G q_{j}^{-(m+\alpha)}}{\sum_{j_{1}=0}^{r_{1}} \cdots \sum_{j_{m}=0}^{r_{m}} G q_{j}^{-(m+\alpha)}}\right] \\
& \tilde{p}_{B L}=-\frac{1}{a} \log \left[\begin{array}{l}
\frac{1}{B\left(\gamma^{*}, \lambda^{*}\right)} \\
\times \int_{0}^{1} \exp (-a p) p^{\left(\gamma^{*}-1\right)}(1-p)^{\left(\lambda^{*}-1\right)} d p
\end{array}\right]
\end{aligned}
$$

where, the integration of Equation 21 is obtained by the numerical integration methods.

General Entropy loss function replacing $u$ by $\theta$ in Equation 19, the Bayes estimate $\tilde{\theta}_{B G}$ of the variable $\theta$ comparative to the general entropy loss function (19) becomes:

$$
\begin{aligned}
& \tilde{\theta}_{B G}=\left[E_{\theta}\left(\theta^{-b} \mid \underline{x}\right)\right]^{(-1 / b)} \\
& =\left[\int_{0}^{\infty} \theta^{-b} \pi_{1}^{*}(\theta \mid x, r) d \theta\right]^{(-1 / b)}
\end{aligned}
$$

And using Equation 25 in (Al-Hossain, 2015b), one can get:

$$
\begin{aligned}
& \tilde{\theta}_{B G}=\left[\frac{\Gamma(m+\alpha-b)}{\Gamma(m+\alpha)}\right]^{(1-/ b)} \\
& \times\left[\frac{\sum_{j_{1}=0}^{r_{1}} \cdots \sum_{j_{m}=0}^{r_{m}} G q_{j}^{-(m+\alpha-b)}}{\sum_{j_{1}=0}^{r_{1}} \cdots \sum_{j_{m}=0}^{r_{m}} G q_{j}^{-(m+\alpha)}}\right]^{(-1 / b)}
\end{aligned}
$$

Replacing also $u$ by $p$ in (19), the Bayes estimate $\tilde{p}_{B G}$ of parameter $p$ relative to the general entropy loss function becomes:

$$
\begin{aligned}
& \tilde{p}_{B G}=\left[\int_{0}^{1} p^{-b} \pi_{2}^{*}(p \mid \underline{X}) d p\right]^{(-1 / b)} \\
& =\left[\frac{\Gamma\left(\gamma^{*}+\lambda^{*}\right) \Gamma\left(\gamma^{*}-b\right)}{\Gamma\left(\gamma^{*}\right) \Gamma\left(\gamma^{*}+\lambda^{*}-b\right)}\right]^{(-1 / b)}
\end{aligned}
$$

\section{Interval Estimation}

Highest Posterior Density Interval (HPDI). In general, the Bayesian procedure to interval estimation is highly more direct than the ML method, for more details (Al-Hossain, 2015b).

\section{Expected Duration}

In many industrial processes, life test is conducted in order to assess the quality of a product. Typically, $n$ products are placed under test and their times to failure are observed. These observed lifetimes are then used to estimate the life distribution of the product. However, in many applications, life tests are usually terminated before the complete lifetimes of $\mathrm{n}$ products are observed. Data from this censored test consist of times to failure of failed units and running times on unfailed units. Following Balakrishnan and Aggrawala (2000), under progressive interval type II censored with random or binomial eliminations conditioning on $R$, the expected the value of $X_{m}$ is presented by (Al-Hossain, 2015b):

$$
\begin{aligned}
& E\left[X_{m} \mid R=r\right] \\
& -2 \theta C(r) \sum_{l_{1}=0}^{r_{1}} \ldots \sum_{l_{m}=0}^{r_{m}}(-1)^{a l} \frac{\left(\begin{array}{c}
r_{i} \\
l_{1}
\end{array}\right) \ldots\left(\begin{array}{c}
r_{m} \\
l_{m}
\end{array}\right)}{\prod_{i=1}^{m-1} h\left(l_{i}\right)} \\
& \times \sum_{k=0}^{\theta h\left(l_{m}\right)-1}(-1)^{k}\left(\begin{array}{c}
\theta h\left(l_{m}\right)-1 \\
k
\end{array}\right)\left(\frac{\sqrt{\pi}}{4(k+1)^{3 / 2}}\right)
\end{aligned}
$$


where, $a l=\sum_{i=1}^{m} l_{i}$ and $h\left(l_{i}\right)=l_{1}+l_{2}+\ldots+l_{i}+i$.

Setting the $r_{i}=0$ for all $i=1, \ldots, m-1$ and $r_{m}=n-m$ in Equation 25, one may get the expected time of a type II censoring test without removals as follows:

$$
\begin{aligned}
& E\left[X_{m}^{*}\right]=2 m \theta\left(\begin{array}{l}
n \\
m
\end{array}\right) \\
& \times \sum_{k=0}^{m \theta-1}(-1)^{k}\left(\begin{array}{c}
m \theta-1 \\
k
\end{array}\right)\left(\frac{\sqrt{\pi}}{4(k+1)^{3 / 2}}\right)
\end{aligned}
$$

By the same way, setting $m=n$ and $r_{i}=0$ for all $i=$ $1, \ldots, m$ in Equation 25, the anticipated time of an entire sampling case with $n$ test units may be written as:

$$
E\left[X_{n}^{* *}\right]=2 n \theta \times \sum_{k=0}^{n \theta-1}\left(\begin{array}{c}
n \theta-1 \\
k
\end{array}\right)\left(\frac{(-1)^{k} \sqrt{\pi}}{4(k+1)^{3 / 2}}\right)
$$

Finally, carrying out the expectation on the life hand side and the right side of Equation 25 with respect to the $R$, the anticipated ending point for gradually type-II censoring with binomial cancellations is estimated from the following equation:

$$
\begin{aligned}
& E\left[X_{m}\right]=E_{R}\left[E\left[X_{m} \mid R=r\right]\right] \\
& =\sum_{r_{1}=0}^{g\left(r_{1}\right)} \cdots \sum_{r_{m-1}=0}^{g\left(r_{m-1}\right)} P(R, p) E\left[X_{m} \mid R=r\right]
\end{aligned}
$$

where, $g\left(r_{1}\right)=n-m, g\left(r_{i}\right)=n-m-r_{1}-\cdots-r_{i-1}, i=2, \cdots, m-1$ and $P(R, p)$ is given in Equation 7 .

The ratio of the expected time under various schemes to the expected time under entire sampling, i.e., Ratio of Expected Experiment Times (REET) is defined in (Al-Hossain, 2015b).

\section{A Simulation Illustration}

\section{Example 1}

In this case, the tests with $n=30,40,60$ and 100 units generated from Burr-X model with variable $\theta$ is examined. The test is finished when the number of failed subjects attains or overtakes a specific value $m$, where $m / n=40,60,80$ and $90 \%$, Data for the dropouts $r_{i}$ were produced from the binomial model as shown in this example. 1000 samples are produced considering the variables $(\theta, p)=(0.321,0.4)$. The MLEs, symmetric Bayes and asymmetric Bayes estimates of $\theta$ and $p$ and their Mean Square Error (MSE) are computed. Considering the results in subsections (3.2) and (4.2), the Approximate Confidence Interval (ACI) in the context of asymptotic distributions of the MLEs and HPDI of the variables are computed and compared in the case of their

\begin{tabular}{|c|c|c|c|c|c|}
\hline \multirow[b]{3}{*}{$\mathrm{n}$} & \multirow[b]{3}{*}{$\mathrm{m}$} & \multirow[b]{3}{*}{ ML } & \multirow[b]{3}{*}{ SE } & \multicolumn{2}{|l|}{ LINEX } \\
\hline & & & & \multicolumn{2}{|l|}{$\mathrm{a}$} \\
\hline & & & & -1 & 1 \\
\hline \multirow[t]{4}{*}{30} & 27 & 0.0645 & 0.0644 & 0.0656 & 0.0633 \\
\hline & 24 & 0.0685 & 0.0683 & 0.0696 & 0.0671 \\
\hline & 18 & 0.0727 & 0.0730 & 0.0747 & 0.0714 \\
\hline & 12 & 0.0785 & 0.0791 & 0.0811 & 0.0773 \\
\hline \multirow[t]{4}{*}{40} & 36 & 0.0573 & 0.0575 & 0.0585 & 0.0566 \\
\hline & 32 & 0.0578 & 0.0580 & 0.0589 & 0.0571 \\
\hline & 24 & 0.0617 & 0.0620 & 0.0631 & 0.0610 \\
\hline & 16 & 0.0657 & 0.0663 & 0.0676 & 0.0651 \\
\hline \multirow[t]{4}{*}{60} & 54 & 0.0455 & 0.0455 & 0.0459 & 0.0451 \\
\hline & 48 & 0.0479 & 0.0480 & 0.0485 & 0.0475 \\
\hline & 36 & 0.0506 & 0.0509 & 0.0516 & 0.0503 \\
\hline & 24 & 0.0528 & 0.0534 & 0.0541 & 0.0526 \\
\hline \multirow[t]{4}{*}{80} & 72 & 0.0400 & 0.0401 & 0.0404 & 0.0398 \\
\hline & 64 & 0.0411 & 0.0413 & 0.0416 & 0.0409 \\
\hline & 48 & 0.0431 & 0.0434 & 0.0438 & 0.0430 \\
\hline & 32 & 0.0479 & 0.0485 & 0.0490 & 0.0479 \\
\hline \multirow[t]{4}{*}{100} & 90 & 0.0338 & 0.0338 & 0.0340 & 0.0337 \\
\hline & 80 & 0.0362 & 0.0363 & 0.0365 & 0.0361 \\
\hline & 60 & 0.0363 & 0.0365 & 0.0367 & 0.0362 \\
\hline & 40 & 0.0399 & 0.0403 & 0.0407 & 0.0400 \\
\hline
\end{tabular}
Coverage Probabilities (CVP). Table 1a-2b show the simulation results for $\theta$ and $\mathrm{p}$, respectively.

Table 1a. The MSE of $\theta$ for $(\theta, p)=(0.321,0.4)$ with CVP of

\begin{tabular}{|c|c|c|c|c|c|}
\hline \multirow[b]{2}{*}{$\mathrm{n}$} & \multirow[b]{2}{*}{$\mathrm{m}$} & \multicolumn{4}{|l|}{ GE } \\
\hline & & $\begin{array}{l}b \\
-1 \\
\end{array}$ & 1 & $\begin{array}{l}\text { CVP } \\
\text { ACI }\end{array}$ & $\begin{array}{l}\text { CVP } \\
\text { HPDI }\end{array}$ \\
\hline \multirow[t]{4}{*}{30} & 27 & 0.0644 & 0.0609 & 0.950 & 0.971 \\
\hline & 24 & 0.0683 & 0.0646 & 0.944 & 0.970 \\
\hline & 18 & 0.0730 & 0.0679 & 0.957 & 0.972 \\
\hline & 12 & 0.0791 & 0.0734 & 0.939 & 0.966 \\
\hline \multirow[t]{4}{*}{40} & 36 & 0.0575 & 0.0542 & 0.956 & 0.965 \\
\hline & 32 & 0.0580 & 0.0550 & 0.958 & 0.976 \\
\hline & 24 & 0.0620 & 0.0589 & 0.941 & 0.971 \\
\hline & 16 & 0.0663 & 0.0626 & 0.945 & 0.966 \\
\hline \multirow[t]{4}{*}{60} & 54 & 0.0455 & 0.0442 & 0.948 & 0.972 \\
\hline & 48 & 0.0480 & 0.0464 & 0.937 & 0.967 \\
\hline & 36 & 0.0509 & 0.0489 & 0.944 & 0.974 \\
\hline & 24 & 0.0534 & 0.0511 & 0.957 & 0.968 \\
\hline \multirow[t]{4}{*}{80} & 72 & 0.0401 & 0.0391 & 0.940 & 0.981 \\
\hline & 64 & 0.0413 & 0.0400 & 0.947 & 0.978 \\
\hline & 48 & 0.0434 & 0.0420 & 0.956 & 0.975 \\
\hline & 32 & 0.0485 & 0.0466 & 0.951 & 0.971 \\
\hline \multirow[t]{4}{*}{100} & 90 & 0.0338 & 0.0332 & 0.951 & 0.977 \\
\hline & 80 & 0.0363 & 0.0356 & 0.941 & 0.972 \\
\hline & 60 & 0.0365 & 0.0357 & 0.961 & 0.986 \\
\hline & 40 & 0.0403 & 0.0391 & 0.952 & 0.985 \\
\hline
\end{tabular}
$95 \% \mathrm{ACI}$ and $95 \% \mathrm{HPDI}$

Table $1 \mathrm{~b}$. The MSE of $\theta$ for $(\theta, p)=(0.321,0.4)$ with CVP of $95 \% \mathrm{ACI}$ and $95 \% \mathrm{HPDI}$ 
Table 2a. The MSE of $\mathrm{p}$ for $(\theta, p)=(0.321,0.4)$. With CVP of $95 \% \mathrm{ACI}$ and $95 \% \mathrm{HPDI}$

\begin{tabular}{|c|c|c|c|c|c|}
\hline \multirow[b]{3}{*}{$\mathrm{n}$} & \multirow[b]{3}{*}{$\mathrm{m}$} & \multirow[b]{3}{*}{ ML } & \multirow[b]{3}{*}{ SE } & \multicolumn{2}{|l|}{ LINEX } \\
\hline & & & & $a$ & \\
\hline & & & & -1 & 1 \\
\hline \multirow[t]{4}{*}{30} & 27 & 0.1713 & 0.1030 & 0.1070 & 0.1001 \\
\hline & 24 & 0.1329 & 0.0995 & 0.1019 & 0.0974 \\
\hline & 18 & 0.1000 & 0.0864 & 0.0877 & 0.0853 \\
\hline & 12 & 0.0766 & 0.0695 & 0.0703 & 0.0683 \\
\hline \multirow[t]{4}{*}{40} & 36 & 0.1571 & 0.1026 & 0.1062 & 0.0997 \\
\hline & 32 & 0.1161 & 0.0937 & 0.0955 & 0.0920 \\
\hline & 24 & 0.0858 & 0.0764 & 0.0774 & 0.0753 \\
\hline & 16 & 0.0653 & 0.0611 & 0.0616 & 0.0607 \\
\hline \multirow[t]{4}{*}{60} & 54 & 0.1368 & 0.1018 & 0.1045 & 0.0994 \\
\hline & 48 & 0.0991 & 0.0854 & 0.0868 & 0.0842 \\
\hline & 36 & 0.0649 & 0.0604 & 0.0610 & 0.0598 \\
\hline & 24 & 0.0527 & 0.0505 & 0.0507 & 0.0502 \\
\hline \multirow[t]{4}{*}{80} & 72 & 0.1232 & 0.0984 & 0.1005 & 0.0964 \\
\hline & 64 & 0.0818 & 0.0737 & 0.0745 & 0.0730 \\
\hline & 48 & 0.0571 & 0.0543 & 0.0546 & 0.0540 \\
\hline & 32 & 0.0455 & 0.0437 & 0.0439 & 0.0435 \\
\hline \multirow[t]{4}{*}{100} & 90 & 0.1102 & 0.0919 & 0.0935 & 0.0904 \\
\hline & 80 & 0.0740 & 0.0682 & 0.0690 & 0.0675 \\
\hline & 60 & 0.0505 & 0.0484 & 0.0487 & 0.0482 \\
\hline & 40 & 0.0423 & 0.0412 & 0.0413 & 0.0410 \\
\hline
\end{tabular}

Table $2 \mathrm{~b}$. The MSE of $p$ for $(\theta, p)=(0.321,0.4)$ with CVP of $95 \% \mathrm{ACI}$ and $95 \% \mathrm{HPDI}$

\begin{tabular}{|c|c|c|c|c|c|}
\hline \multirow[b]{3}{*}{$\mathrm{n}$} & \multirow[b]{3}{*}{$\mathrm{m}$} & \multicolumn{2}{|l|}{ GE } & \multirow[b]{2}{*}{ CVP } & \multirow[b]{2}{*}{ CVP } \\
\hline & & $b$ & & & \\
\hline & & -1 & 1 & ACI & HPD \\
\hline \multirow[t]{4}{*}{30} & 27 & 0.1030 & 0.1140 & 0.973 & 0.982 \\
\hline & 24 & 0.0995 & 0.1006 & 0.936 & 0.978 \\
\hline & 18 & 0.0864 & 0.0857 & 0.928 & 0.966 \\
\hline & 12 & 0.0695 & 0.0685 & 0.953 & 0.982 \\
\hline \multirow[t]{4}{*}{40} & 36 & 0.1026 & 0.1026 & 0.922 & 0.982 \\
\hline & 32 & 0.0937 & 0.0937 & 0.958 & 0.979 \\
\hline & 24 & 0.0764 & 0.0747 & 0.942 & 0.972 \\
\hline & 16 & 0.0611 & 0.0607 & 0.952 & 0.984 \\
\hline \multirow[t]{4}{*}{60} & 54 & 0.1018 & 0.1012 & 0.937 & 0.976 \\
\hline & 48 & 0.0854 & 0.0842 & 0.930 & 0.963 \\
\hline & 36 & 0.0604 & 0.0596 & 0.956 & 0.981 \\
\hline & 24 & 0.0505 & 0.0504 & 0.950 & 0.982 \\
\hline \multirow[t]{4}{*}{80} & 72 & 0.0984 & 0.0966 & 0.945 & 0.983 \\
\hline & 64 & 0.0737 & 0.0734 & 0.943 & 0.977 \\
\hline & 48 & 0.0543 & 0.0539 & 0.952 & 0.975 \\
\hline & 32 & 0.0437 & 0.0434 & 0.952 & 0.978 \\
\hline \multirow[t]{4}{*}{100} & 90 & 0.0919 & 0.0907 & 0.928 & 0.955 \\
\hline & 80 & 0.0682 & 0.0673 & 0.940 & 0.980 \\
\hline & 60 & 0.0484 & 0.0481 & 0.948 & 0.976 \\
\hline & 40 & 0.0412 & 0.0410 & 0.941 & 0.965 \\
\hline
\end{tabular}

Table 3a. a Presents $\mathrm{E}\left[X_{m}\right]$ for type-II when $\theta=1$

\begin{tabular}{llllll}
\hline $\mathrm{n}$ & $\mathrm{m}$ & $\mathrm{p}=0.1$ & $\mathrm{p}=0.2$ & $\mathrm{p}=0.3$ & $\mathrm{p}=0.4$ \\
\hline 6 & 6 & 1.5203 & 1.5203 & 1.5203 & 1.5203 \\
& 5 & 1.2537 & 1.3162 & 1.3618 & 1.3941 \\
& 4 & 1.0043 & 1.0635 & 1.1235 & 1.1825 \\
& 3 & 0.7925 & 0.8365 & 0.8844 & 0.9367 \\
10 & 10 & 1.6757 & 1.6757 & 1.6757 & 1.6757 \\
& 9 & 1.5057 & 1.5771 & 1.6114 & 1.6271 \\
& 8 & 1.3358 & 1.4549 & 1.5256 & 1.5631 \\
& 7 & 1.1586 & 1.2927 & 1.4013 & 1.4719 \\
& 6 & 1.0060 & 1.1082 & 1.1981 & 1.2714 \\
& 5 & 0.8667 & 0.9684 & 1.0799 & 1.1854 \\
15 & 15 & 1.7914 & 1.7914 & 1.7914 & 1.7914 \\
& 14 & 1.4617 & 1.6105 & 1.7098 & 1.7521 \\
& 13 & 1.2286 & 1.4340 & 1.6046 & 1.6928 \\
& 12 & 1.1335 & 1.3766 & 1.5755 & 1.6725 \\
& 11 & 1.0939 & 1.3261 & 1.5268 & 1.6313 \\
& 10 & 0.9943 & 1.0772 & 1.2417 & 1.3968 \\
& 9 & 0.9039 & 0.9929 & 1.1738 & 1.3372 \\
& 8 & 0.6164 & 0.7063 & 0.9518 & 1.2154 \\
& 7 & 0.5360 & 0.5979 & 0.8036 & 1.0596 \\
\hline
\end{tabular}

Table 3b. Presents $E\left[X_{m}\right]$ for type-II when $\theta=1$

\begin{tabular}{lllll}
\hline $\mathrm{p}=0.5$ & $\mathrm{p}=0.6$ & $\mathrm{p}=0.7$ & $\mathrm{p}=0.8$ & $\mathrm{p}=0.9$ \\
\hline 1.5203 & 1.5203 & 1.5203 & 1.5203 & 1.5203 \\
1.4160 & 1.4304 & 1.4394 & 1.4449 & 1.4482 \\
1.2363 & 1.2810 & 1.3144 & 1.3366 & 1.3498 \\
0.9931 & 1.0514 & 1.1080 & 1.1580 & 1.1970 \\
1.6757 & 1.6757 & 1.6757 & 1.6757 & 1.6757 \\
1.6341 & 1.6373 & 1.6390 & 1.6399 & 1.6406 \\
1.5817 & 1.5908 & 1.5955 & 1.5981 & 1.5999 \\
1.5114 & 1.5317 & 1.5421 & 1.5479 & 1.5516 \\
1.3305 & 1.3809 & 1.4259 & 1.4637 & 1.4884 \\
1.2719 & 1.3346 & 1.3757 & 1.4009 & 1.4161 \\
1.7914 & 1.7914 & 1.7914 & 1.7914 & 1.7914 \\
1.7657 & 1.7692 & 1.7700 & 1.7704 & 1.7706 \\
1.7288 & 1.7416 & 1.7458 & 1.7473 & 1.7479 \\
1.7072 & 1.7174 & 1.7204 & 1.7219 & 1.7228 \\
1.6724 & 1.6862 & 1.6910 & 1.6934 & 1.6950 \\
1.5140 & 1.5909 & 1.6344 & 1.6552 & 1.6631 \\
1.4513 & 1.5268 & 1.5781 & 1.6109 & 1.6264 \\
1.4048 & 1.5085 & 1.5565 & 1.5772 & 1.5859 \\
1.2787 & 1.4213 & 1.4943 & 1.5245 & 1.5362 \\
\hline
\end{tabular}

From the above tables which give the simulation results, we may conclude that:

- The ML technique gives preferable assessments of $\theta$, it means that it has smaller MSE, whereas the Bayes assessments of $p$ getting smaller MSE

- The MSE accompanying with both MLE and Bayes assessments of the variables go down with increasing the specimen size $n$. In addition, it diminishes while the percentage of censoring becomes small (i.e., $m$ becomes large)

- The procedure considers highest posterior density accomplishes better than the asymptotic normality 
based on a method of obtaining closer coverage probability to the normal value

- In general, we remark that the MSEs of all estimates of the variables $\theta$ and $\mathrm{p}$ become smaller while the elimination probability $p$ is large

\section{Example 2}

Example 2 calculates the anticipated test time $E\left[X_{m}\right]$ for the different parameters $n, m, p$ and $\theta$ numerically based on Type-II progressive censoring with binomial eliminations. We examined some values like: $n=6,10$, $15, \theta=1,2,3$ and $p=0.1, \cdots, 0.9$. The results are presented in Table 3a-4b with remarking that when $m=$ $n$ be in agreement with the complete sample plan.

Table 4. (a) Presents E $\left[X_{m}\right]$ for type-II when $\theta=2$

\begin{tabular}{llllll}
\hline $\mathrm{n}$ & $\mathrm{m}$ & $\mathrm{p}=0.1$ & $\mathrm{p}=0.2$ & $\mathrm{p}=0.3$ & $\mathrm{p}=0.4$ \\
\hline 6 & 6 & 1.7285 & 1.7285 & 1.7285 & 1.7285 \\
& 5 & 1.4870 & 1.5436 & 1.5850 & 1.6142 \\
& 4 & 1.2592 & 1.3092 & 1.3617 & 1.4154 \\
& 3 & 1.0678 & 1.1067 & 1.1485 & 1.1945 \\
10 & 10 & 1.8698 & 1.8698 & 1.8698 & 1.8698 \\
& 9 & 1.7149 & 1.7800 & 1.8112 & 1.8255 \\
& 8 & 1.5611 & 1.6691 & 1.7333 & 1.7674 \\
& 7 & 1.9374 & 1.5178 & 1.6179 & 1.6836 \\
& 6 & 1.2609 & 1.3457 & 1.4200 & 1.4824 \\
& 5 & 1.1370 & 1.2286 & 1.3290 & 1.4244 \\
15 & 15 & 1.9754 & 1.9754 & 1.9754 & 1.9754 \\
& 14 & 1.6289 & 1.7832 & 1.8904 & 1.9365 \\
& 13 & 1.3870 & 1.5967 & 1.7800 & 1.8759 \\
& 12 & 1.2989 & 1.5442 & 1.7553 & 1.8596 \\
& 11 & 1.2767 & 1.5032 & 1.7114 & 1.8216 \\
& 10 & 1.1895 & 1.2416 & 1.4048 & 1.5688 \\
& 9 & 1.1087 & 1.1662 & 1.3440 & 1.5137 \\
& 8 & 0.7774 & 0.8479 & 1.1056 & 1.3891 \\
& 7 & 0.6990 & 0.7406 & 0.9559 & 1.2306 \\
\hline
\end{tabular}

Table $4 \mathrm{~b}$. Presents $E\left[X_{m}\right]$ for type-II when $\theta=2$

\begin{tabular}{lllll}
\hline $\mathrm{p}=0.5$ & $\mathrm{p}=0.6$ & $\mathrm{p}=0.7$ & $\mathrm{p}=0.8$ & $\mathrm{p}=0.9$ \\
\hline 1.7285 & 1.7285 & 1.7285 & 1.7285 & 1.7285 \\
1.6341 & 1.6472 & 1.6553 & 1.6603 & 1.6632 \\
1.4659 & 1.5086 & 1.5407 & 1.5618 & 1.5742 \\
1.2451 & 1.2987 & 1.3516 & 1.3988 & 1.4354 \\
1.8698 & 1.8698 & 1.8698 & 1.8698 & 1.8698 \\
1.8319 & 1.8348 & 1.8363 & 1.8372 & 1.8378 \\
1.7843 & 1.7925 & 1.7968 & 1.7992 & 1.8008 \\
1.7202 & 1.7388 & 1.7483 & 1.7536 & 1.7570 \\
1.5359 & 1.5851 & 1.6317 & 1.6721 & 1.6987 \\
1.5030 & 1.5600 & 1.5975 & 1.6204 & 1.6342 \\
1.9754 & 1.9754 & 1.9754 & 1.9754 & 1.9754 \\
1.9512 & 1.9550 & 1.9558 & 1.9562 & 1.9564 \\
1.9152 & 1.9291 & 1.9336 & 1.9350 & 1.9356 \\
1.8969 & 1.9075 & 1.9105 & 1.9118 & 1.9127 \\
1.8648 & 1.8790 & 1.8837 & 1.8859 & 1.8873 \\
1.6956 & 1.7797 & 1.8273 & 1.8499 & 1.8581 \\
1.6350 & 1.7164 & 1.7723 & 1.8080 & 1.8245 \\
1.5942 & 1.7064 & 1.7578 & 1.7795 & 1.7880 \\
1.4674 & 1.6217 & 1.7002 & 1.7316 & 1.7430 \\
\hline
\end{tabular}

From Table $3 a-5 b$, it is noticed that the anticipated of ending time for Type-II cumulative censoring sample is getting close to that of the entire sample when $\mathrm{m}$ is getting bigger. For a constant value of $\mathrm{m}$, the expected experiment time of type-II cumulative with binomial eliminations goes down while the sample size $\mathrm{n}$ getting bigger. Also, with respect to binomial eliminations, it is obvious that for constant values of $n$ and $m$, the REET becoming close to one faster for going up of $\mathrm{p}$. All results are because of the fact that a high cancellation of probability shows a large number of dropouts. Therefore, the cancellation of the probability $p$ plays a considerable factor in the time needed to accomplish the experiment. In all cases, a large number of test units $n$ would abbreviate the experiment period of the test while the underlying schedule is Type-II with binomial eliminations.

Table 5. (a) Presents $\mathrm{E}\left[X_{m}\right]$ for typ-II when $\theta=3$

\begin{tabular}{llllll}
\hline $\mathrm{n}$ & $\mathrm{m}$ & $\mathrm{p}=0.1$ & $\mathrm{p}=0.2$ & $\mathrm{p}=0.3$ & $\mathrm{p}=0.4$ \\
\hline 6 & 6 & 1.8414 & 1.8414 & 1.8414 & 1.8414 \\
& 5 & 1.6128 & 1.6664 & 1.7055 & 1.7332 \\
& 4 & 1.3967 & 1.4418 & 1.4903 & 1.5412 \\
10 & 3 & 1.2179 & 1.2538 & 1.2921 & 1.3345 \\
& 10 & 1.9754 & 1.9754 & 1.9754 & 1.9754 \\
& 9 & 1.8283 & 1.8901 & 1.9198 & 1.9333 \\
& 8 & 1.6826 & 1.7849 & 1.8458 & 1.8782 \\
& 7 & 1.5260 & 1.6391 & 1.7350 & 1.7982 \\
& 6 & 1.3982 & 1.4737 & 1.5396 & 1.5963 \\
15 & 5 & 1.2832 & 1.3690 & 1.4633 & 1.5533 \\
& 15 & 2.0761 & 2.0761 & 2.0761 & 2.0761 \\
& 14 & 1.7197 & 1.8774 & 1.9890 & 2.0372 \\
& 13 & 1.4741 & 1.6856 & 1.8758 & 1.9760 \\
& 12 & 1.3849 & 1.6350 & 1.8533 & 1.9616 \\
& 11 & 1.3838 & 1.5997 & 1.8119 & 1.9251 \\
& 10 & 1.2752 & 1.3294 & 1.4933 & 1.6622 \\
& 9 & 1.2490 & 1.2610 & 1.4362 & 1.6094 \\
& 8 & 0.8360 & 0.9230 & 1.1885 & 1.4831 \\
& 7 & 0.8176 & 0.8190 & 1.0380 & 1.3229 \\
\hline
\end{tabular}

Table $5 \mathrm{~b}$. Presents $E\left[X_{m}\right]$ for type-II when $\theta=3$

\begin{tabular}{lllll}
\hline $\mathrm{p}=0.5$ & $\mathrm{p}=0.6$ & $\mathrm{p}=0.7$ & $\mathrm{p}=0.8$ & $\mathrm{p}=0.9$ \\
\hline 1.8414 & 1.8414 & 1.8414 & 1.8414 & 1.8414 \\
1.7520 & 1.7644 & 1.7721 & 1.7768 & 1.7796 \\
1.5899 & 1.6316 & 1.6630 & 1.6836 & 1.6954 \\
1.3817 & 1.4326 & 1.4835 & 1.5291 & 1.5643 \\
1.9754 & 1.9754 & 1.9754 & 1.9754 & 1.9754 \\
1.9394 & 1.9422 & 1.9436 & 1.9445 & 1.9450 \\
1.8942 & 1.9021 & 1.9061 & 1.9084 & 1.9099 \\
1.8333 & 1.8511 & 1.8601 & 1.8652 & 1.8683 \\
1.6469 & 1.6956 & 1.7431 & 1.7850 & 1.8126 \\
1.6277 & 1.6818 & 1.7173 & 1.7390 & 1.7521 \\
2.0761 & 2.0761 & 2.0761 & 2.0761 & 2.0761 \\
2.0526 & 2.0564 & 2.0573 & 2.0576 & 2.0579 \\
2.0170 & 2.0315 & 2.0361 & 2.0375 & 2.0381 \\
2.0003 & 2.0112 & 2.0142 & 2.0154 & 2.0163 \\
1.9696 & 1.9840 & 1.9886 & 1.9907 & 1.9921 \\
1.7944 & 1.8824 & 1.9322 & 1.9558 & 1.9643 \\
1.7347 & 1.8194 & 1.8778 & 1.9151 & 1.9323 \\
1.6969 & 1.8137 & 1.8671 & 1.8892 & 1.8978 \\
1.5695 & 1.7303 & 1.8118 & 1.8439 & 1.8550 \\
\hline
\end{tabular}




\section{Conclusion}

The problem of estimating the parameters of Burr distribution type- $X$ based on a mean square error while data are gathered in the context of Type-II censoring with binomial eliminations are considered. Interval and both point assessments of the variables and the anticipated time to accomplish the test has been computed and interpreted for various censoring schedules. The outcomes illustrate that the maximum likelihood estimation for various estimators of the variable is going down while the cancellation probability $\mathrm{p}$ becoming large, furthermore, the corresponding time needed to accomplish the test going up significantly. A numerical example is given to illustrate the procedures and the accuracy of prediction intervals is investigated via Monte Carlo simulation. Furthermore, this study shows how simulation can be a helpful and illuminating way to approach problems in Bayesian analysis. Moreover, the simulation has presented some interesting properties of the Bayes estimates. After an extensive study of results. Bayesian problems of updating estimates can be handled easily and straight forwardly with simulation. Since we can express the distribution function of the Burr Type- $X$ as well as its inverse in closed form, the inversion method of simulation is straightforward to implement. The study can further be extended by considering generalized versions of the distribution under the variety of circumstances.

\section{Acknowledgment}

The author is grateful to the referees for their helpful suggestions that improved this article.

\section{Ethics}

This article is original and contains unpublished material. In the same time I confirm that no ethical issues are involved.

\section{References}

Ahmad, A.A., M.E. El-Adll and T.A. ALOafi, 2015. Estimation under Burr type $\mathrm{X}$ distribution based on doubly type II censored sample of dual generalized order statistics. J. Egypt. Math. Society, 23: 391-396. DOI: 10.1016/j.joems.2014.03.011

Al-Hossain, A.Y., 2015a. Characterizations for the power inverse Gaussian and generalized inverse Gaussian distributions based on conditional moments. J. Math. Stat., 11: 70-74. DOI: 10.3844/jmssp.2015.70.74

Al-Hossain, A.Y., 2015b. Estimation for Burr-X model based on progressively censored with random removals: Bayesian and non-Bayesian Proceedings of the Conference on Approaches. Mathematical Methods and Systems in Science and Engineering, Jan. 10-12, Tenerife, Canary Islands, Spain, pp: 100-107.
Al-Hossain, A.Y., 2016. Predictive inference from the Exponentiated Weibull model given adaptive progressive censored data. Applied Math. Inf. Sci., 10: 1177-1184.

AL-Hussaini, E.K., A.H. Abdelhamid and A.F. Hashem, 2014. Bayesian prediction intervals of order statistics based on progressively type-II censored competing risks data from the half-logistic distribution. J. Egypt. Math. Society, 23: 190-196. DOI: 10.1016/j.joems.2014.01.008

Ali, S., 2014. Mixture of the inverse Rayleigh distribution: Properties and estimation in a Bayesian framework. Applied Math. Modell., 39: 515-530. DOI: 10.1016/j.apm.2014.05.039

Amin, Z.H., 2008. Bayesian inference for the Pareto lifetime model under progressive censoring with binomial removals. J. Applied Stat., 35: 1203-1217. DOI: $10.1080 / 09537280802187634$

Balakrishnan, N. and R. Aggarwala, 2000. Progressive Censoring: Theory, Methods and Applications. 1st Edn., Birkhäuser, Boston, ISBN-10: 0817640010, pp: 248.

Balakrishnan, N. and R.A. Sandu, 1995. A simple simulational algorithm for generating progressive Type-II censored samples. Am. Stat., 49: 229-230. DOI: $10.1080 / 00031305.1995 .10476150$

Balakrishnan, N., N. Knnan, C.T. Lin and H. Ng, 2003. Point and interval estimation for Gaussian distribution based on Progressively Type-II censored samples. IEEE Trans. Reliab., 52: 90-95. DOI: $10.1109 /$ TR.2002.805786

Banerjee, A. and D. Kundu, 2008. Inference based on Type-II hybrid censored data from a Weibull distribution. IEEE Trans. Reliab., 57: 369-378. DOI: 10.1109/TR.2008.916890

Clifford, C.W.G., I. Mareschal, Y. Otsuka and T.L. Watson, 2015. A Bayesian approach to person perception. Consciousness Cognit., 36: 406-413. DOI: $10.1016 /$ j.concog.2015.03.015

Cohen, A.C., 1963. Progressively censored samples in life testing. Technometrics, 5: 327-329. DOI: $10.1080 / 00401706.1963 .10490102$

Efron, B., 1982. The bootstrap and other resampling plans. Proceedings of the CBMS-NSF Regional Conference Series in Applied Mathematics, (SAM' 82), SIAM, Philadelphia, PA., pp: 38-38.

Fernandez, A.J., 2004. On estimating exponential parameters with general type II progressive censoring. J. Statist. Plann. Inference, 121: 135-147. DOI: $10.1016 / \mathrm{S} 0378-3758(03) 00083-1$

Karam, N.S. and A.K. Jbur, 2014. Bayesian analyses of the Burr type $\mathrm{X}$ distribution under doubly type II censored samples using different priors and loss functions. Math. Theory Model., 4: 9-18. 
$\mathrm{Ku}$, C. and M.F. Kaya, 2007. Estimation for the parameters of the Pareto distribution under progressive censoring. Comm. Statist. Theory Meth., 36: 1359-1365.

DOI: $10.1080 / 03610920601077089$

Mahmoud, M.A.W., A.A. Soliman, A.H. Abd-Ellah and R.M. El-Sagheer, 2013. Bayesian inference and prediction using progressive first-failure censored from generalized Pareto distribution. J. Stat. Applied Pro., 2: 269-279. DOI: 10.12785/jsap/020310

Naranjo, L., J. Martn and C.J. Perez, 2014. Bayesian binary regression with exponential power link. Comput. Stat. Data Anal., 71: 464-476. DOI: $10.1016 /$ j.csda.2012.07.022

Ng, H.K., P.S. Chan and N. Balakrishnan, 2004. Optimal progressive censoring plans for the Weibull distribution, Technometrics, 46: 470-481. DOI: $10.1198 / 004017004000000482$

Okasha, H.M., 2014. E-Bayesian estimation for the Lomax distribution based on type-II censored data. J. Egypt. Math. Society, 22: 489-495. DOI: 10.1016/j.joems.2013.12.009

Okasha, M.K. and M.Y. Matter, 2015. On the threeparameter burr type xii distribution and its application to heavy tailed lifetime data. J. Adv. Math., 10 3428-3442.

Pathak, A. and A. Chaturvedi, 2014. Estimation of the reliability function for two-parameter exponentiated Rayleigh or Burr type X distribution. Stat. Optimiz. Inform. Comput., 2: 305-322.

Silva, R.B. and G.M. Cordeiro, 2015. The Burr XII power series distributions: A new compounding family. Braz. J. Probab. Stat., 29: 565-589. DOI: $10.1214 / 13-B J P S 234$

Soliman, A.A., 2005. Estimation of parameters of life from progressively censored data using Burr-XII model. IEEE Trans. Reliab., 54: 34-42. DOI: $10.1109 /$ TR.2004.842528

Soliman, A.A., A.H. Abd Ellah, N.A. Abou-Elheggag and A.A. Modhesh, 2011. Bayesian inference and prediction of Burr type-XII distribution for progressive first failure censored sampling. Intell. Inform. Manage., 3: 175-185.

DOI: $10.4236 /$ iim.2011.35021
Sutikno, H.K. and L.D. Ratih, 2014. Gaussian Copula Marginal regression for modeling extreme data with application. J. Math. Stat., 10: 192-200.

DOI: $10.3844 /$ jmssp.2014.192.200

Torney, D.C., 2005. Bayesian analysis of binary sequences. J. Computat. Applied Math., 175: 231-243. DOI: 10.1016/j.cam.2004.05.010

Tse, S.K., C. Yang and H.K. Yuen, 2000. Statistical analysis of Weibull distributed lifetime data under type II progressive censoring with binomial removals. J. Applied Stat., 27: 1033-1043. DOI: 10.1080/02664760050173355

Wang, F.K. and C.W. Lee, 2014. M-estimator for estimating the Burr type III parameters with outliers. Math. Comput. Simulat., 105: 144-159. DOI: $10.1016 /$ j.matcom.2014.05.010

Wu, S.J., 2003. Estimation for the two-parameter Pareto distribution under progressive censoring with uniform removals. J. Stat. Comput. Simul., 73: 125-134. DOI: 10.1080/00949650215732

Wu, S.J., Y.J. Chen and C.T. Chang, 2007. Statistical inference based on progressively censored samples with random removals from the Burr type XII distribution. J. Statist. Comput. Simulat., 77: 19-27. DOI: 10.1080/10629360600569204

Yan, L., F. Yang and C. Fu, 2009. A Bayesian inference approach to identify a Robin coefficient in onedimensional parabolic problems, J. Computat. Applied Math., 231: 840-850. DOI: $10.1016 /$ j.cam.2009.05.007

Zellner, A., 1995. Bayesian and non-Bayesian approaches to statistical inference and decisionmaking. J. Computat. Applied Math., 64: 3-10. DOI: $10.1016 / 0377-0427(95) 00002-\mathrm{X}$ 